\title{
SIGNIFICANCE OF EQ IN STRESS REDUCTION \& COPING
}

\author{
Uma Warrier* Aisha. M. Sheriff**
}

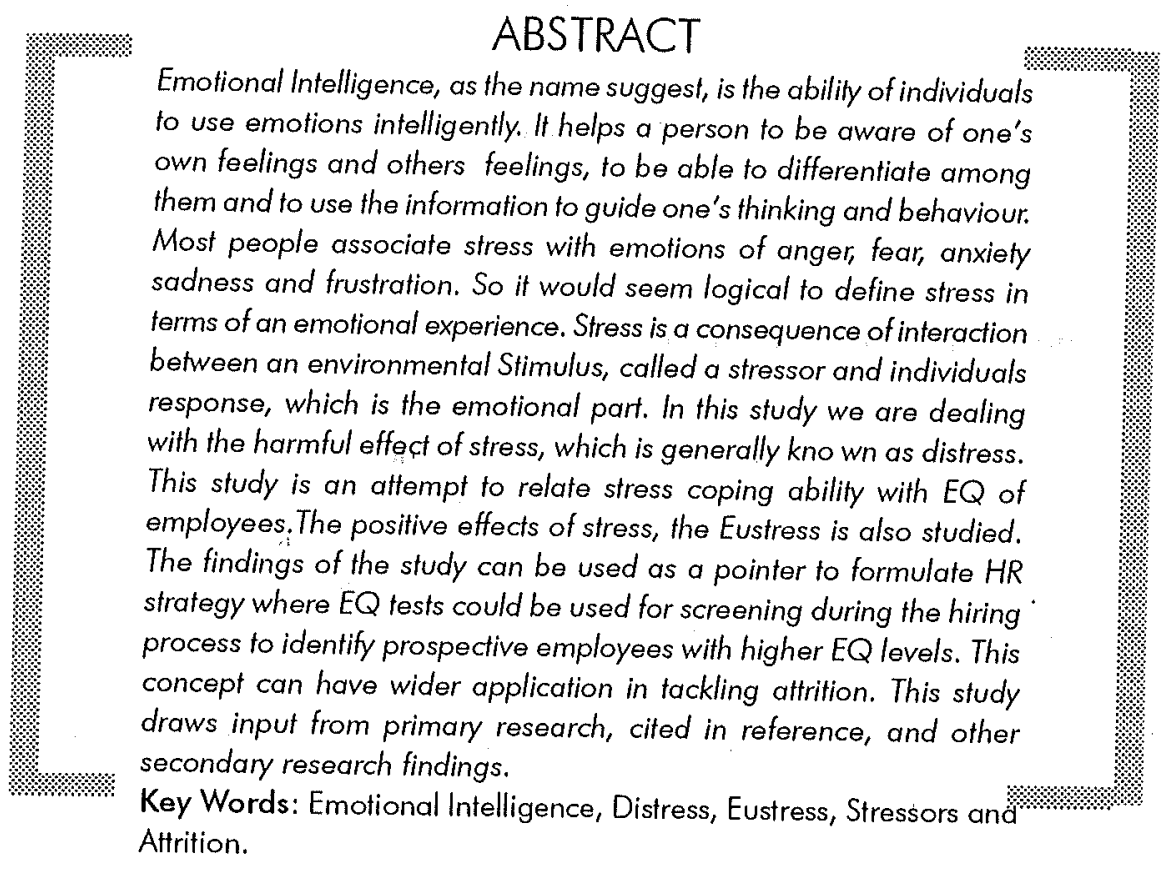

* Lecturer, Center for Management Studies, Sri Bhagwan Mahaveer Jain College, Bangalore umawarrier@rediffmail.com

** Assistant Professor, S.J.C.E, Mysore, aishasheriff@hotmail.com 


\section{Introduction}

As long ago as 1987, one of the most prominent researchers in the field of stress, Richard Lazarus, wrote:

"Although we have usually referred to stress, coping theory and research, we think that we should now speakless of stress and more of emotion. Stress, which primarily concerns negative person-environment relationships, cognitive appraisals and emotional response states such as fear, anger, guilt and shame, falls under the larger rubric of emotion."

(Lazarus \& Folkman, 1987) 71

The word 'stress' is defined by the Oxford Dictionary as "a state of affair involving demand on physical or mental energy". A condition or circumstance (not always adverse), which can disturb the normal physical and mental health of an individual. In medical parlance 'stress' is defined as a perturbation of the body's homeostasis. This demand on mind-body occurs when it tries to cope with incessant changes in life. A 'stress' condition seems 'relative' in nature. Extreme stress conditions, psychologists say, are detrimental to human health but in moderation stress is normal and, in many cases, proves useful. Stress can be good or bad. Accordingly, it can be classified as:

\section{Stress: Eustress and Distress}

As long as we think of stress as negative, we will try to move it away from us, keep it at arm's length or try to prevent having stress in the first place. In fact, stress is a life energy force in the body and is the key ingredient in optimal performance. Positive stress is actually essential and beneficial, and can be our strongestally for leading stimulating healthy lives, filled with vitality and resilience.

When unregulated, uncontrolled or ignored, stress can be harmful to your health and wellbeing; too little stress can lead to apathy, atrophy, fatigue and illness. A major step toward successful stress management is to find and maintain a personal equilibrium, a balance that works for you at each stage of your life. 


\section{Positive Role of Stress}

Although "stress" has become synonymous with something bad, such as traffic jams and too many work demands, some stress is good for you. Pioneering stress researcher Hans Selye differentiated between damaging stress (distress) and positive stress (eustress). Positive stress can inspire us to do our best and to perform better than if we were under no stress. Psychologists tell us that our level of performance is directly related to the level of stress we experience. This theory says that low and high levels of arousal decrease performance, whereas intermediate levels enhance performance. In other words, we are capable of peak levels of performance when under a moderate amount of stress.

High

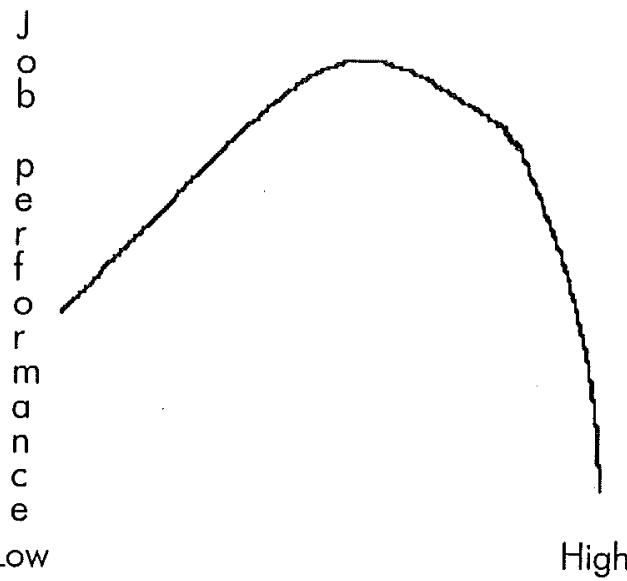

There are three types of negative stress: physical, emotional, and mental stress.

Physical Stress: Physical stress is the result of doing too much with body in a given amount of time. It's working too hard. It's not getting enough rest.

It's using substances which are harmful to it. Such substances include caffeine, alcohol, sugar, tobacco, and food additives. Environmental considerations come in here too, such as dust, smoke and exposure to the fumes of chemicals and solvents etc. Loud industrial noise is very fatiguing and stressful to the body as well.

These are all forms of physical stress which contribute to weakening the immune system. 
Emotional Stress: There are many different emotions. Happiness and joy do not contribute to a weaker immune system. But anger, resentment, worry, and fear do. These emotions can cause sleep loss, which can contribute to physical stress too.

And what produces emotion? Remember, an emotion is a feeling. And what we think, influences how we react to certain events or people. This in turn determines the emotion we will be feeling. Positive, happy thoughts are what we want for a strong immune system.

Mental Stress: Mental stress results from too much work to the mental faculty without adequate rest or relaxation. Mental stress can also result from working on ambiguous data, conflicting but compelling information, decision making under uncertainty.

\section{Physical Effects of Stress}

The symptoms manifested due to excessive stress are Head ache, High Blood Pressure, Heart Disease, Arthritis, Asthma, Diabetes, Stomach ulcers, Migraines, Poor appetite, Nausea, Dizziness, Eczema etc.

\section{Psychological Effects of stress}

Stress can cause a change in behaviour of a person. The psychological effects of stress are Anger, Frustration, Sleeplessness, Tension, Irritation, Anxiety, Tiredness, Low self esteem Depression, Dissatisfaction with life, Suicidal tendencies, Heavy smoking, Drug abuse etc

\section{Major Causes of stress}

The causes for stress could be either intrinsic or due to external environmental factors. Some of the intrinsic factors that cause stress are personality Type A, Being highly ambitious, and extrinsic factors like Irregularity of food habits, Work overload, Poor relationship with the boss, colleagues or subordinates, Lack of autonomy, Role Ambiguity, Role conflict, Hectic lifestyle Job insecurity, Poor pay, Physical demands of the job, Intrinsic impoverishment of the job etc. 


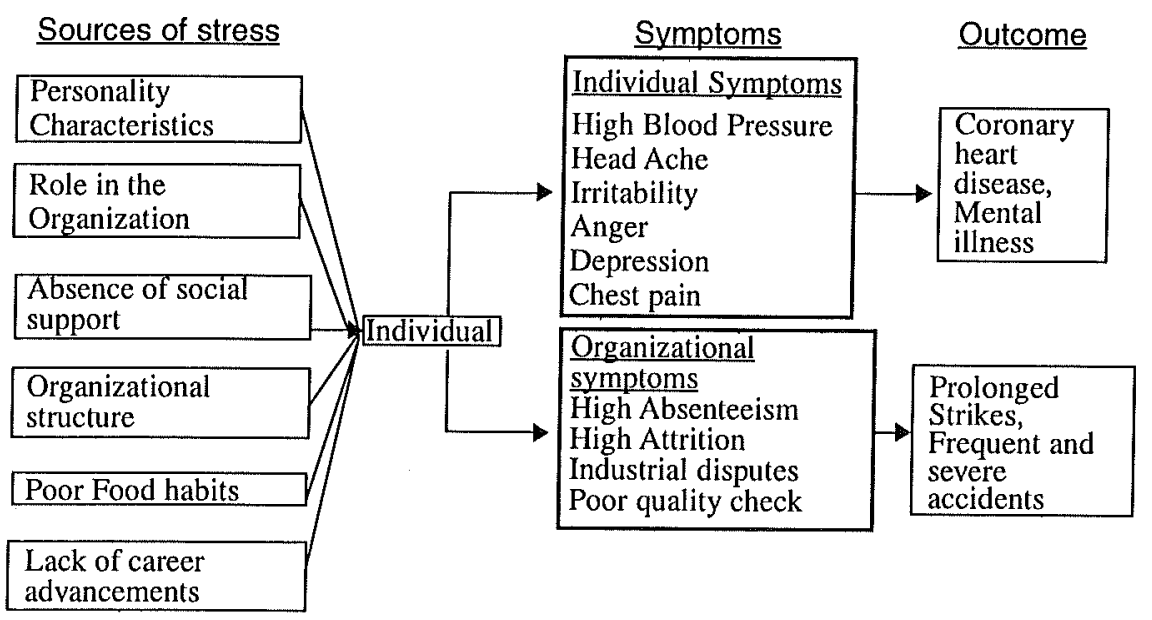

As we have seen earlier, stress at adequate level is good for an individual's growth. When stress goes beyond the adequate level, one needs to moderate it. Physical stress is easy to understand and manage. However, mental and emotional stress is difficult to understand and manage. One needs to understand the cause of such stress and also know the ways and means of managing such stress. Emotional Intelligence provides set of framework to understand such stress levels and helps oneself to manage and cope up with such stress.

\section{What is Emotional Intelligence?}

Emotional Intelligence, as the name suggest, is the ability of individuals to use emotions intelligently. It helps a person to be aware of one's own feelings and others feelings, to be able to differentiate among them and to use the information to guide one's thinking and behaviour. El indicates set of two major competencies which can help an individual to understand oneself better and others as well. 
El Framework

\begin{tabular}{|c|c|}
\hline Personal Competence & Social Competence \\
\hline SELF - AWARENESS & SOCIAL AWARENESS \\
\hline $\begin{array}{l}\text { Emotional awareness } \\
\text { Accurate self-assessment } \\
\text { Self-confidence }\end{array}$ & $\begin{array}{l}\text { Empathy } \\
\text { Service orientation } \\
\text { Developing others } \\
\text { Leveraging diversity } \\
\text { Political awareness }\end{array}$ \\
\hline SELF \pm REGULATION & SOCIAL SKILLS \\
\hline $\begin{array}{l}\text { Self-control } \\
\text { Trustworthiness } \\
\text { Conscientiousness } \\
\text { Adaptability } \\
\text { Innovativeness }\end{array}$ & $\begin{array}{l}\text { Influence } \\
\text { Communication } \\
\text { Leadership } \\
\text { Change catalyst } \\
\text { Conflict management } \\
\text { Building bonds } \\
\text { Collaboration and cooperation } \\
\text { Team capabilities }\end{array}$ \\
\hline SELF \pm MOTIVATION & \\
\hline $\begin{array}{l}\text { Achievement drive } \\
\text { Commitment } \\
\text { Initiative } \\
\text { Optimism }\end{array}$ & \\
\hline
\end{tabular}

\section{Evolution of El Study}

The early Emotional Intelligence theory was originally developed during the 1970's and 80's by the work and writings of psychologists Howard Gardner (Harvard), Peter Salovey (Yale) and John Mayer (New Hampshire). It draws on psychological and organizational theories. Goleman (1998) proposed that high levels of El were associated with success in a business context. It is suggested that "emotionally intelligent" individuals can perceive, 
understand and regulate the emotions of others, thus making emotional intelligence a significant factor in the success of interpersonal interaction in a work context (Mayer et al., 2000). In exploring this construct Bar-On (2000) saw emotional intelligence as being a multifunctional array of interrelated emotional, personal and social abilities, which influence our overall ability to actively and effectively cope with demands and pressures.

$E Q$ is related to many of the organizational aspects like Organizational commitment, job security, work relationship, communication, organizational stress efc. Of these, stress is one of the important aspects. Since stress is conceived mainly as an emotional reaction (usually negative) to various environmental stimuli, (Selye, 1956), EQ could be used as a framework, within which the individual could learn how to cope up with it and how to control strong emotions.

Often, researchers define stress as the psychological and physiological conditions that a person experiences when they perceive a situation as stressful. This means that whenever we experience a change in our environment, we may experience stress, but what is stressful for one person might not be for another. Many factors influence how you interpret events including:

- Who are you

- Your attitudes

- Your skills

- Your personality

- The current situation

- Other events in your life

In one of the very few studies exploring the impact of EQ on stress coping, Slaski and Cartwright (2002) has found that managers with high EQ suffered less subjective stress and had better physical and physiological well being.

In India, especially with regard to Information Technology (IT) industry, there is good scope for a study relating EQ and Stress, because of the fact that the employees of IT Industry generally have long working hours, short time for meeting the deadlines, and high targets to meet. All these factors could lead to organizational stress. Since the concept of EQ is contemporary 
and has much scope to research on the relation between EQ and Stress in work situation, this was considered as an ideal topic for study.

\section{Ways of coping with stress- The Emotional Intelligence way!}

As we know, we have five parameters of emotional intelligence and manipulating these variables can augment the Emotional Quotient of individuals. An increased EQ has a positive impact on stress coping styles of individuals. Let's look at the possibilities of increasing each component of $E Q$ by simple practices.

\section{For Improving Self awareness:}

A training session using Johari Window will help an individual to understand oneself from the perspective of self and others. Assertive training will help in saying NO when we have to say NO. Reduced perfectionism is another outcome of improved self awareness. Perfectionists generally succumb to stress very easily. An awareness that a person is tending to be a perfectionist can help him to tone down his desire to live up to almost impossible standards. Prioritise your work, which will give you a breather. Increased self awareness can also bring in better tolerance to ambiguity.

\section{For improving Self motivation:}

Increase the hardiness- which is a triad of $3 C_{s}$, control, commitment and challenge. This can help you sail through the problem. Develop optimistic outlook towards life and practice positive thinking. Calculated risk taking where you are sure of achieving the benchmark is one sure way to motivate self. Some tips on Time management will help you to organize your life, which will motivate you to do things in a better way. Avoid people who invalidate you. Be in a group of people who are enthusiastic.

\section{For improving Self control:}

Training on anxiety management is an efficient way to control anxiety and thereby better self control. Techniques to control anger like taking deep breath, counting from 1 to 20 backwards etc could be adapted to improve self control. Relaxation techniques and practicing yoga are very well known techniques of stress reduction, which actually are the tools for improving 
self control. Listening to soothing music, repeating a peaceful "Mantra", focusing on a pleasant mental image etc can go a long way in improving self control.

\section{For Improving Social skills:}

Managers should practice open channels of communication for better organizational climate. Build a work culture to reduce negative feelings.

Leadership training using managerial grid will help to understand the kind of task or person orientation of leaders can help them to tone down the extremes. Training on transactional analysis will help individuals to have better understanding on different ego states we all have and the different kinds of transactions occur when we communicate. This will reduce a lot of conflicts, which arise out of misunderstanding. Clear understanding of kinds of "strokes" we give to and receive from others will help in improving interpersonal relationships in the work place. Understanding the different life positions and choosing the "I am OK, you are OK" life position will bring in better harmony in the organization, there by reducing stress levels of employees. One golden rule of influencing others is "Never fall short of praises!" Further, a collaborative approach to conflict resolution can enhance team spirit making the workplace congenial to work.

\section{For Improving Social Awareness:}

Identifying the kind of internal groups in an organization and identifying its members will help in making intelligent decisions regarding formal group formation. Listen twice as much as you speak, because you have two ears and just one tongue! Use humour to reduce stress levels. Delegate work based on expertise. Learn to empathize with other people. Extend social support for each other. Providing professional counselor's service in each organization will help in de-stressing

\section{Secondary literature review}

2.1 An article was presented by loannis Tsaousis, University of Aegena, Greece, and loannis Nikolaou, Athens university of Economics and business, Greece (International Journal of organizational analysis, 2002,Vol.10, No. 4, pp 327-342), "Emotional Intelligence at work place: Exploring its effect on occupational stress and organizational commitment" 
They have conducted a study by administering EQ questionnaire and an Organisational Stress Screening Tool (ASSET). The results were in the expected direction, showing a negative correlation between emotional intelligence and stress at work place.

2.2 Another study conducted by Ronald J.Bureke, Faculty of Administrative studies, York University, Ontario, Canada, titled "Stress, Work \& Professional satisfaction and militancy among Canadian Physicians" (Journal Of Organizational Behaviour, Vol. 14, 459-472,1993). Data was collected from 2087 men \& women physicians using questionnaire. He could identify relationship between stress at work place \& Professional satisfaction, which is closely related to $E Q$.

2.3 A study by Arie Shirom \& Ada Maye (Journal of Organizational behavior, September 1993, Vol 14 Number 5), Identified that increased stress among working people will increase their vulnerability to psychological strain, which has an inverse relation with $E Q$.

2.4 Michael Rock Ed.D, a certified EQ coach and facilitator, who has more than 150 articles, books and $C D$ s to his credit has published an article on "Stress, EQ and Unstress you", in "Canada One" online magazine. $\mathrm{He}$ is of the opinion that Stress tolerance and Impulse control are the 2 basic parameters to measure $E Q$, which is in accordance with Baron, who created a measure for finding $E Q$ level of people.

\section{Research Design And Methodology}

An exploratory research was undertaken to formulate the problem and develop hypothesis. Literature survey was conducted at the Libraries of Indian Institute of Management (IIM), Bangalore and Mount Carmel college of Management, Bangalore. Further discussions with HR Managers of different IT Companies were made to reconfirm the feasibility and applicability of the study in work situations. From the above exploratory work, it was evident that $\mathrm{EQ}$ is gaining popularity among practitioners and theorists of management. Since organizational stress and strain pose threat to efficiency of the employees and hence the profitability of the company, stress is a very important parameter, which needs attent ion. The respondents covered in this study are the Middle and Top Level managers of Aditi 
Technologies and Accenture. Analytical research is conducted, trying to explore the relationship between stress levels of employees and their $E Q$. Simple random sampling is used. Sample size of 70 is taken for the study. Primary data was obtained through fieldwork by administering the interview schedule to the top and middle level managers of Aditi Technologies and Accenture. Secondary data was collected from review of previous literature, journals, books, magazines, and Internet.

\section{Objectives of the study}

- To identify critical organizational factors which contribute to high levels of stress.

- To measure factors causing stress in the work places.

- To measure the $E Q$ levels of various employees.

- To find the correlation between stress and EQ levels of Employees.

\section{Hypothesis:}

- The Null Hypothesis (Ho): - There exists no relationship between Stress and $E Q$.

- Alternate Hypothesis (Hi): -There is a correlation between Stress and EQ.

\section{Analysis of data and results:}

The data collected was tabulated and condensed using arithmetic mean. In order to ascertain the relationship between the stress and EQ parameter, correlation analysis was conducted with the help of Karl Pearson's Coefficient of Correlation, Hypothesis testing was done using Karl Pearson's Correlation Coefficient to identify the nature of relationship between the two variables, $E Q$ and Stress.

The null hypothesis was that "There exist no relation between EQ and Stress" against an alternative hypothesis of "There exists a relation between Stress \& EQ". 
Karl Pearson's Coefficient of correlation $(r)=\frac{\left.\sum(X-\bar{X}), Y-\bar{Y}\right)}{N \sigma_{z} \sigma_{y}}$

Karl Pearson's Correlation coefficient was calculated using the above equation and found to be -0.65 . From the correlation analysis the relation between Stress \& EQ was found out to be negative. With higher values of $E Q$ among people, stress level was found to be less and the people with less $E Q$ were found to be highly stressed.

One of the primary objectives of the study was to identify a relation between Stress at work place \& EQ level of the employees of two Software firms, Aditi Technologies \& Accenture. From the study conducted with the help of Questionnaires \& interviews, along with supporting secondary data, it could be concluded that there is a relation between Stress \& EQ at work place.

Statistical analysis was done with the help of correlation to identify the nature of relation between the 2 variables, Stress \& EQ.A negative value of $r=-.6$ shows that there is a negative correlation between Stress \& EQ. This finding gives us ample scope to reject the null hypothesis that there is No relation between EQ \& Stress.

From the data collected \& analyzed, it was evident that Role clarity was not a factor leading to stress in both the organisations, Aditi Technologies \& Accenture.

Role overload is identified as a stressor for employees of both the companies, Aditi Technologies\& Accenture. The pressure is on completion of job within targeted time Role conflict is medium to low among employees of Aditi Technologies \& Accenture. They feel that they can strike a good balance between personal \& professional life.

Majority of the respondents opined that there is no stress due to political pressure as they were not forced to do things which are of not their interest $\&$ which violate company policy.

From the table showing poor peer relation, which is one of the stressor, was identified to be low. So poor peer relationship do not cause stress among employees of these 2 companies 
Increasing physical demands of the job was identified as a stress factor by both the companies' employees. They feel that their jobs really demand a lot of their time \& energy.

Role Ambiguity is not considered as a stress factor by both the company employees. They feel that their superiors do not give contradicting orders. Intrinsic impoverishment was found to be very high among employee of both the organisation. This means that the employees feel that there is enough scope for growth \& advancement in the current job. So intrinsic impoverishment is not a stress factor in both companies Aditi Technologies \& Accenture. Employees from the IT industry being knowledge workers yearn for opportunities to learn and grow.

Quality of work life was considered as another stress parameter to check the extent of stress among employees. It was found that they were provided with good canteen facility, seating arrangement, good lighting \& ventilation within the office premises. Hence Quality of work life is not a causative stress factor in both the companies, Aditi Technologies \& Accenture.

Employees of both the companies believed that they were under rewarded which was found to be a stress factor among them. They felt that they are underpaid as compared to the quantum of work executed. This leads to stress in work place.

The EQ Parameter on Self Regulation was found to be low among both the company's employees.

Employees of both the companies, Aditi Technologies\&. Accenture were found to be having moderate to high score on Empathy.

The EQ parameter on Self adaptability was found to be moderate among the employees of Aditi Technologies\& Accenture which is indicative of the fact that more attention needs be paid to personal change by its employees. The last EQ Parameter, Self awareness was found to be poor among the employees of Aditi Technologies\& Accenture. From the correlation analysis the relation between Stress \& EQ was found out to be negative. With higher values of $E Q$ among people, stress level was found to be less and the people with less EQ were found to be highly stressed. 


\section{Suggestions for better coping with stress:}

Emotional intelligence training programs, which are well established \& practiced, can be adopted. As one of the job stressor identified was nonprofitability of the job, the company can take a cue from this \& enhance the package to make the employees feel that they paid enough in comparison to the quantum of the job.

Since the EQ Parameter Self regulation shows a lower score, Self regulation techniques like training on anxiety management, organising Yoga sessions for the employees, arranging for counseling sessions etc.

Since the EQ parameter on Self awareness is found to be low, a workshop on Johari Window model will help the employees to learn a lot about self from his own \& others perspective.

\section{Managers must keep open channels of communication}

Companies should continuously look out for signs \& symptoms of Burnout, which are ongoing employee frustration, sleep deprivation, short temperament, cynicism etc

Build a work Culture which minimizes helplessness \& hopelessness. Stress arising out of strenuousness of work can be managed by providing various organisational programs like employee Wellness program, which focuses on employees overall mental \& physical health. Providing employees the best recreational facilities within the company premises \& frequent breaks can reduce strenuousness. Stress caused due to role overload can be reduced by educating the employees about the various techniques of coping with stress like Meditation, Aerobics, Yoga etc.

\section{References}

\section{Books}

1. Goleman, Daniel," Emotional intelligence", Bantam Books, Boston.

2. Goleman, Daniel," Working with Emotional intelligence" Bantam Books, Boston. 
3. Steve Ellis \& Penny Dick, Introduction to organisational behaviour, Mc Graw Hill International edition, Singapore, 1998.

\section{Working Paper}

1. B.Dholakia \& Geethika Kaushal," Emotional intelligence at work place" TAA Communication area, Indian Institute of Management, Ahmedbad, (Working paper no. 2001-05-01, Page no 2 to 15).

2. D.M Pestonjee \& Prabhat Kumar Mishra, "Role stress \& Job Satisfaction Among Doctors". Organization behaviour Area, Indian Institute of Management, Ahmedabad (Working paper No 98-09-04)

3. Q.N. Heky, "Emotional intelligence, Capability \& Radical change", working paper A-1089, INSEAD, France.

\section{Journal}

1. PVL Raju "All About Emotional Intelligence", ICFAl Press, March 2004, page 15.

2. Loannis Tsaousis", Emotional Intelligence at work place: Exploring its effect on occupational stress and organizational commitment", . The international Journal of organizational analysis, (2002, Vol.10, No 4, pp.299-301).

3. K. Harigopal and R.Ravikumar "Stress at work place", Indian Journal of Applied Psychology, (1979,16, (2), 61- 67.

4. Dick, P. (2000) "The social construction of meaning of acute stressors: a qualitative study of the personal accounts of police officers using a stress counseling service", Work \& Stress, Vol 14 (3), pages 226-244.

5. Hart, K. (1990) "Introducing Stress \& Stress Management to Managers", Journal of Managerial Psychology, Vol. 5, pages 9-16.

6. Eileen Berlin Ray \& Katherine Miller (1994)."Journal of Applied Behavioural Science, Vo I. 30, No. 3, September 1994, 357-373.

\section{Websites}

1. www.eiconsortium.org 